\title{
SIMPLE BAND MODEL OF DOPED FULLERENE CRYSTAL
}

\author{
A. KOPER AND M. MUCHA \\ Institute of Physics, Adam Mickiewicz University \\ Umultowska 85, 61-614 Poznań, Poland
}

We use quantum billiard with many scattering centers to describe conducting electrons properties in $\mathrm{A}_{n} \mathrm{C}_{60}$ crystals, where $\mathrm{A}$ denotes alkali metal. We focus our attention on the $\mathrm{A}_{3} \mathrm{C}_{60}$ crystal, for which we calculate the band structure, density of states, and conductivity for normal electrons. Conductivity shows linear dependence on temperature in this model, which agrees well with experimental data. We also discuss consequences of our results for superconductivity mechanism in $\mathrm{A}_{3} \mathrm{C}_{60}$ and possibilities of analogous approach to describe electron properties in fused fullerenes and multiply connected carbon clusters.

PACS numbers: 74.20.Mn, 74.25.Kc, 74.25.Jb

\section{Introduction}

Although the band structure of $\mathrm{A}_{n} \mathrm{C}_{60}$ crystals is relatively well known (cf. [1-3]) there are still some doubts about applicability of conventional Fermi liquid theory (in low temperatures) to these crystals (cf. [4]). Experiments show that for a wide range of temperatures $T$ the resistivity depends linearly on $T$, particularly in $\mathrm{A}_{3} \mathrm{C}_{60}$ (cf. [5]), which cannot be attributed to scattering on classical phonons. For the same reason status of superconductivity phonon mechanism is unclear in these crystals [4]. Thus it is important to investigate consequences of simple models, where the mechanism of electrons bands structure appearance in $\mathrm{A}_{n} \mathrm{C}_{60}$ crystals and interaction of electrons with lattice vibrations is considered. In this paper we use as such a model three-dimensional quantum billiard, where free electrons scatter on $\mathrm{C}_{60}$ molecules (the scattering centers) located in lattice nodes. The scattering centers are modeled by potential $V(x)$ of an isolated $\mathrm{C}_{60}$ molecule. It can be characterized as spherically symmetric attracting well $V(x)$ (cf. [1]). Bottom of the well is a sphere of radius $R=3.55 \AA$ and its depth is $\Delta R=3.0 \AA$. The alkali ions $\mathrm{A}$ in $\mathrm{A}_{n} \mathrm{C}_{60}$ crystals modify symmetry and range of the potential $V(x)$ by polarizing the electrons in fully occupied molecular band $h_{\mathrm{u}}$. They are located in high symmetry points in the inter nodes gaps (cf. e.g. [6]). Free electrons move between $\mathrm{C}_{60}$ molecules mainly between overlapping potential wells (distance $\mathrm{C}_{60}-\mathrm{C}_{60}$ is of order $\approx 10 \AA$, cf. [1]): 
In this paper we show that the model of quantum billiard in $\mathrm{A}_{n} \mathrm{C}_{6}$ crystals, in spite of its simplicity, is powerful enough to produce basic features of the band structure. We show that in this model free electrons tend to occupy three overlapping (or lying very close one to another) bands. In order to maintain consistency with $\mathrm{C}_{60}$ molecule spectral structure we shall refer to these bands as $t_{1 \mathrm{u}}$. We use the band structure obtained within this model to determine resistivity of $\mathrm{A}_{3} \mathrm{C}_{60}$ crystal. In our calculations we use the Kubo-Greenwood formula.

\section{The band structure (quantum billiard model)}

We shall focus our attention on $\mathrm{A}_{3} \mathrm{C}_{60}$ crystal. It has fcc structure with $\mathrm{C}_{60}$ located at lattice nodes. The $\mathrm{A}$ ions occupy all available interstitial positions. That is why in first approximation we can assume that the spherical symmetry of potential $V(x)$ is preserved.

Since we make only a model calculation we shall replace skew elementary cell of fcc lattice with simple cubic one having a lattice constant $a=10 \AA$, which is equal to real distance between the nearest neighbors $\mathrm{C}_{60}-\mathrm{C}_{60}$ in fcc lattice. Value of the potential $V_{B}$ inside the spherical well is negative. In numerical calculations we take $V_{B}=-4 \mathrm{eV}$. The parameters $V_{B}, a, R, \Delta R$ determine the bands structure.

In our calculations we should also take into account the geometry (G) of the $\mathrm{C}_{60}$ molecule. The simplest way to do this is by expressing diameter $D=7.1 \AA$ of the molecule in terms of energy $V_{\mathrm{G}}=3 \mathrm{eV}$. The electrons with this energy will be the most effectively scattered by $\mathrm{C}_{60}$ centers.

Consequently we get the following formula for our spherical potential well model of the quantum billiard:

$$
V(x)=V_{B}\left\{\theta\left[(R+\Delta R)^{2}-x^{2}\right]-\theta\left(R^{2}-x^{2}\right)\right\}+V_{\mathrm{G}} \theta\left(R^{2}-x^{2}\right),
$$

where $\theta$ is the Heaviside step function. The Fourier transform of this potential in arbitrary dimension $d$ is expressed by the Bessel $J_{d / 2}$ functions. This property allows for relatively easy numerical calculations of electron's Bloch states $\psi_{\nu, k}(x)$ and their energies $E_{\nu, k}$ (in the $\nu$-th band for a vector $k$ from the Brillouin zone) from the Schrödinger equation

$$
\left[-\frac{\hbar^{2}}{2 m} \nabla^{2}+\sum_{i} V\left(x-\boldsymbol{R}_{i}\right)\right] \psi_{\nu, \boldsymbol{k}}(x)=E_{\nu}(k) \psi_{\nu, k}(x),
$$

where $V\left(\boldsymbol{x}-\boldsymbol{R}_{i}\right)$ denotes potential around $i$-th lattice node, whose location is indicated by vector $\boldsymbol{R}_{i}$ and $m$ is the electron mass. The sum runs over all sc lattice nodes. In the case of finite crystals of volume we assume periodic boundary conditions. One can easily notice that for dimension $d=1$ potential $V(x)$ becomes the Kronig-Penny model.

\section{Resistivity (mass operator and vertex operator)}

In the case of partially filled band $t_{1 \mathrm{u}}$ the electrons from a neighborhood of the Fermi surface will scatter on phonons and themselves. In our model we can describe all kinds of vibrations which occur in real system. They can be accounted for by considering distortions of balls surfaces, i.e. actually displacements 
of spheres $\theta\left[(R+\Delta R)^{2}-\left(\boldsymbol{x}-\boldsymbol{R}_{i}\right)^{2}\right]$ or $\theta\left[R^{2}-\left(x-\boldsymbol{R}_{i}\right)^{2}\right]$, which can be regarded as a spherical membrane. Here we will use only the transversal "breathing" vibrations, which seem to interact with electrons most effectively. Vibrations tangent to the spheres or locally perpendicular vibrations are less effective for obvious reasons. The breathing vibrations of internal ball are a reflection of the same vibrations of carbon atoms in $\mathrm{C}_{60}$ while the breathing vibrations of external ball result from breathing vibrations of $\mathrm{A}$ ions. The internal vibrations can be neglected in first approximation since the total mass of carbon atoms in core $\mathrm{C}_{60}$ is very large. Therefore we remain with the external vibrations. Their phonon energy is of order $E_{\mathrm{A}} \approx 100 \mathrm{~cm}^{-1}$. We shall denote the function $\sum_{i} V_{B} \theta\left[\left(R+\Delta R+r_{i}\right)^{2}-\left(\boldsymbol{x}-\boldsymbol{R}_{i}\right)^{2}\right]$ as $V_{b}(r, x)$ or shortly $V_{b}$. The parameters $r=\left(r_{i}\right)$ describe transversal displacements of external sphere. As it is commonly known (cf. [7]) in order to calculate resistivity from the Kubo-Greenwood formula it is necessary to derive the mass operator $\Sigma$ and the vertex operator $\Gamma$. Here we calculate them in the first order of the non-crossing approximation

$$
\Sigma=\left\langle V_{\mathrm{m}} \frac{1}{E-H_{0}} V_{\mathrm{m}}\right\rangle_{\mathrm{ph}}, \quad \Gamma=\left\langle V_{\mathrm{m}} \hat{p} V_{\mathrm{m}}\right\rangle_{\mathrm{ph}},
$$

where ph denotes mean value of phonon fluctuations over temperature, $H_{0}$ is Hamiltonian (2), where the function $V_{b}$ is replaced with $\left\langle V_{b}\right\rangle_{\mathrm{ph}}, \hat{\mathrm{p}}$ is momentum operator, $V_{\mathrm{m}}=V_{b}-\left\langle V_{b}\right\rangle_{\mathrm{ph}}$. This shows that significant contribution to $\Sigma$ and $\Gamma$ comes from membrane potential $V_{\mathrm{m}}$. Calculation of mean values in (3) can be done explicitly, since it consists in calculating of iterated Gaussian integrals over variables $r=\left(r_{i}\right)$, where the square of standard deviation $\sigma_{\mathrm{A}}^{2}=\hbar^{2}\left(2 m_{\mathrm{A}} E_{\mathrm{A}}\right)^{-1}$ $\operatorname{coth}\left(\beta E_{\mathrm{A}} / 2\right)$ and $m_{\mathrm{A}}$ is $\mathrm{A}$ ion mass. The $\sigma_{\mathrm{A}}^{2}$ is small for real systems. For potassium atom in temperature $T=0 \mathrm{~K}$ it is of order $4.3 \times 10^{-4} \AA^{2}$, and thus it can be used for series expansion. Therefore our approximation for mass and vertex operators seems to be sufficiently justified.

\section{Results and conclusions}

Results of our calculations are presented in Fig. 1. It is clearly visible that the bands $t_{1 \mathrm{u}}$ constitute a group of three bands. Degree of overlapping and their width depends strongly on the depth of the well $V_{B}$. The deeper the well the more overlapped and narrower the bands are. In real $\mathrm{A}_{3} \mathrm{C}_{60}$ system the width is of order $\approx 0.5 \mathrm{eV}$. In our model this occurs for $V_{B} \approx-6.0 \mathrm{eV}$. In the density of states graph we can see $t_{1 \mathrm{~g}}$ and higher bands. The geometrical parameter $V_{\mathrm{G}}$ has insignificant influence on the spectral structure of the system.

Graph of resistivity $\rho(T)$ as function of temperature, in temperature range 100-350 K, shows nearly linear dependence. This result was obtained by taking into account interactions not with "point-like" object but with extended objects resembling spherical membranes. In this context it confirms the statement that scattering on phonons should not lead to linear dependence [4]. It would be interesting to investigate the "membrane mechanism" of electron pairing (in contrast to phonon one) as well as influence of the quantum chaos on superconductivity for the billiard model.

Because of its simplicity the model can also be used for description of electron properties in fused fullerenes and multiply connected carbon clusters [8]. In the 

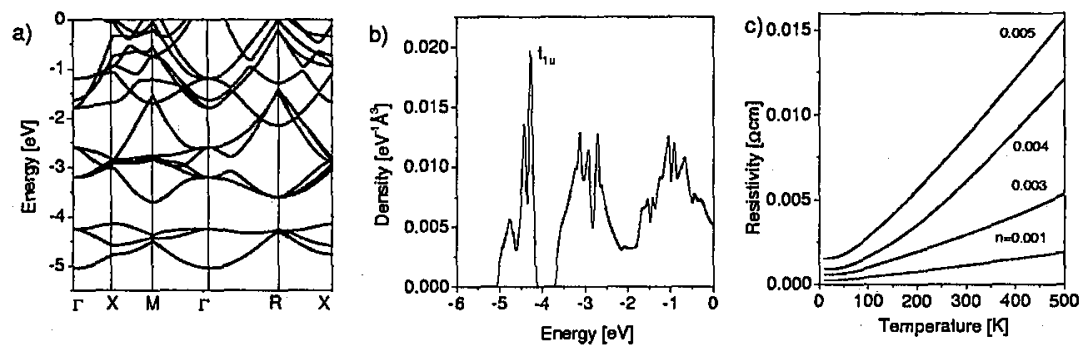

Fig. 1. Selected properties of quantum billiard model for $A_{3} C_{60}$ crystal. (a) The bands electron structure. (b) Density of states $g(E)=2(2 \pi)^{-3} \int_{\mathrm{BZ}} \sum_{\nu} \delta\left(E-E_{\nu}(k)\right)$ graph. The Shrödinger equation was solved numerically for $5 \times 5 \times 5$ cluster in the reciprocal lattice. (c) Graph illustrating dependence of $\rho(T)$ on temperature $T$ for different densities of electrons measured in units of $10^{21} \mathrm{~cm}^{-3}$.

case of these systems the potential $V(x)$ will have symmetry implied by the surface geometry of clusters.

\section{References}

[1] N. Troullier, J.L. Martins, Phys. Rev. B 46, 1754, 1766 (1992).

[2] S.C. Erwin, in: Buckminsterfullerenes, Eds. W.E. Billups, M.A. Ciufolini, VCH Publishers, New York 1993, p. 217.

[3] A.A. Remova, V.P. Shpakov, U. Paek, V.R. Belosludov, Phys. Rev. B 52, 13715 (1995).

[4] V.J. Emery, S.A. Kivelson, V.N. Mathukumar, preprint available on http://xxx.lanl.gov; cond-mat/9901270.

[5] W.A. Vareka, A. Zettel, Phys. Rev. Lett. 72, 4121 (1994).

[6] R.C. Haddon, Acc. Chem. Res. 25, 127 (1992).

[7] E.N. Economou, Green's Functions in Quantum Physics, Springer-Verlag, Berlin 1983.

[8] M. Menon, E. Richter, K.R. Subaswamy, Phys. Rev. B 57, 4063 (1998). 